\title{
The effect of $\boldsymbol{\beta}$-cyclodextrin on the Formation of Phospholipid Micelles: A Molecular Dynamics Simulation Study
}

\author{
D. MAKIEŁA $^{a, *}$, K. GÓRnY ${ }^{a}$ And Z. GBurski ${ }^{a, b}$ \\ ${ }^{a}$ Institute of Physics, Silesian Centre for Education and Interdisciplinary Research, \\ University of Silesia in Katowice, Poland \\ ${ }^{b}$ Katowice Institute of Information Technologies, Mickiewicza 29, 40-085 Katowice, Poland \\ (Received July 19, 2019; revised version August 12, 2019; in final form August 21, 2019)
}

\begin{abstract}
Using the molecular dynamics simulation method we studied the aqueous solution of $\beta$-cyclodextrin and 1,2-dimyristoyl-sn-glycero-3phosphocholine (DMPC) molecules. We observed that in water environment DMPC molecules form a "core" surrounded by $\beta$-cyclodextrins which stick to the surface of DMPC core. The developed $\beta$-cyclodextrin shell (corona) might facilitate the transport of DMPC cluster in bioenvironment. Moreover some smaller binary complexes, composed of only single pair of $\beta$-cyclodextrin and DMPC were detected. Our simulations showed that $\beta$-cyclodextrin do not influence the structure of lipid aggregates and should not negatively interfere the liposomal drug delivery systems.
\end{abstract}

DOI: 10.12693/APhysPolA.136.542

PACS/topics: Molecular dynamics, Phospholipids, Oligosacharides, Computer simulation

\section{Introduction}

Lipids are one of the most common type of biomolecules and are stored in almost every living cell. They are the main building block of biomembranes, participating in cell energy storage and transmission of nerve signals. Due to their frequent occurrence in the mammal cells, their physical properties, and their role in carcinogenesis, the lipids can be utilized in cancer diagnosis by means of Raman imaging [1-3]. Phospholipid molecules are an essential component of the membrane of biological cells. Their amphiphilic properties i.e. both hydrophilic and lipophilic, lead to the formation of a lipid bilayera characteristic element of biomembrane. The phospholipid molecule consists a hydrophilic "head" (phosphate group) and two hydrophobic fatty acids called "tails" of the phospholipide. It is a glycerol molecule that joins together these two building blocks, the hydrophilic "head" and hydrophobic "tails". Simulation studies have shown, that phospholipids have a strong affinity for silicon-carbide and carbon nanotubes, forming thin layers on their surfaces [4-6].

Some types of molecules undergo self-association under specific conditions to form aggregates such as micelles, chains, vesicles, layers [7-9]. In case of some amphipathic molecules, like DMSO, it was shown that the hydrophilic interactions are enchanced by temperature, leading to the solubility of substances in polar solvents [10]. The geometry of aggregates formed by am-

\footnotetext{
* corresponding author; e-mail damian.makiela@smcebi.edu.pl
}

phipathic molecules is a result of a subtle balance between two opposing forces. The attractive hydrophobic interaction contribute to the aggregation of surfactant molecules, whereas the electrostatic repulsion between the polar, hydrophilic parts determines the lower size that an aggregate can attain.

The phospholipid micelle is spherical in shape with its hydrophilic part ("head") exposed to external medium. The hydrocarbon part of phospholipid (hydrophobic "tail") is directed towards the micelle center. The various kind of micelles have been the subject of scientific research for years. Also, their applications in pharmaceutical, cosmetic, and food industries were developed.

Recently, the molecular dynamics (MD) simulations have become an effective method to study various micelles [11-21]. The important asset of MD method is its ability to simulate phenomena at the atomic level. In this research we have used MD simulation method to investigate thoroughly the effect of $\beta$-cyclodextrin on the formation of phospholipid micelles composed of the important cell membrane constituent, namely, 1,2-dimyristoyl-sn-glycero3phosphocholine (DMPC) molecules.

The $\beta$-cyclodextrin $(\beta$-CD) is oligosaccharide supramolecule with a unique toroidal (cupshaped) structure. It is composed of seven D-glucose units bonded with $\alpha$-1,4-glysosidic linkage. The depth of the $\beta \mathrm{CD}$ pocket equals 7.8 , while its width varies from 7.8 (top) to 5.7 (bottom). The well-known and appreciate property of $\beta \mathrm{CD}$ molecule is a high biocompatibility and good water-solubility. A stereochemical arrangement of the toroidal shape $\beta$-CD molecule implies that its interior is hydrophobic, while the external part of $\beta$-CD is hydrophilic. This stereochemical formation of $\beta$-cyclodextrin enables the complexation of some 
small enough hydrophobic molecules in $\beta$-CD inert cavity. That way, the host-guest complexes are formed. In consequence, one observes both the water solubility and the overall biocompatibility of the formed systems. Our research also showed that $\beta$-CD increases the solubility of cholesterol in an aqueous environment [22]. The excellent biocompatibility and unique inclusion capability as well as powerful functionalization capacity of cyclodextrins and their derivatives make them especially attractive for engineering novel functional materials for biomedical applications [23-27]. Lipids can be encapsulated by means of complexation with $\beta$-CD or its derivatives. The interaction between the lipids and cyclodextrins and its derivatives is not only defined by the size of the cyclodextrin inner cavity but also by the chemical groups surrounding entrance to the cavity. The question naturally arises whether $\beta$-CD and its derivatives can be utilized to extract specific lipid types from the bio-member anes or micelles [28]. This suggestive idea has been the subject of several experimental studies, for example via NMR spectroscopy [29, 30], fluorescence microscopy [31], neutron scattering [32], Brewster microscopy [33], calorimetry [34]. Here, we incorporate the computer simulation technique to the study of this issue. In the context of developing novel targeted drug delivery techniques, further studying the interactions between $\beta$-CDs and phospholipids like DMPC is of special interest. $\beta$-CD can be utilized to increase the solubility of the liposomal agregates or to faciliate their opening. Our results have implications for the interpretation of experimental measurements, and may help in the rational design of efficient CD based nano-carriers. There has been increasing interest recently to fabricate supramolecular systems for drug and gene delivery based on cyclodextrin materials [35-38].

\section{Simulation details}

The MD simulations were performed using NAMD 2.11 [39] simulation software with the allatom CHARMM force field [40] and visualized with VMD 1.9.2 [41]. The studied systems were composed of $9 \beta$-CD and $\mathrm{n}=9,18,45$ DMPC molecules in water environment. The periodic boundary conditions were applied. The structure of $\beta \mathrm{CD}$ [42] molecule is shown in Fig. 1.

The initial simulation box was set to $100 \times 100 \times 100 \AA^{3}$. The number of water molecules packed in this box was: 30850,30208 , and 28400 for $\beta$ CD:DMPC ratios 1:1, 1:2 and 1:5, respectively. In these simulation runs pressure was controlled using Langevin barostat. The reference pressure was set to 1 atm. The Langevin piston was used to control the pressure at $1 \mathrm{~atm}$, with a piston period of $100 \mathrm{fs}$, a damping time constant of $50 \mathrm{fs}$. Long-range electrostatic forces were taken into account by means of the Paricle Mesh Ewald [43] approach. The equilibration in NPT (constant number of particles, constant pressure, and

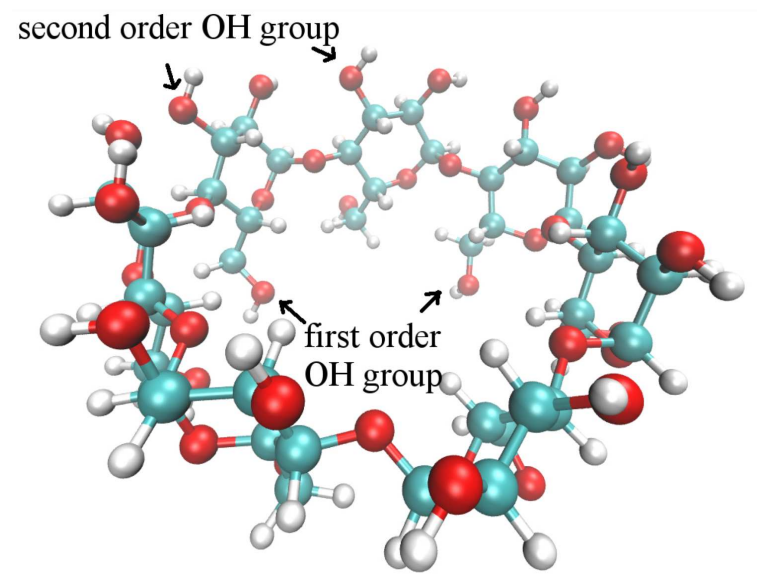

Fig. 1. The structure of $\beta$ cyclodextrin-VMD visualization.

constant temperature) ensemble for aqueous was performed. The integration time step was set to $\Delta t=1.0 \mathrm{fs}$ for all simulation runs. The standard NAMD integrator (Brünger-Brooks-Karplus algorithm) [44] was used. Next, the "production" phases were started. The duration was 50 ns for bulk samples with water. During this stage the data were collected every 2000 simulation steps (2 ps). The systems were simulated at six temperatures $(T=280,290,300,310,320$, and $330 \mathrm{~K})$. The temperature was maintained, employing Langevin thermostat with a damping coefficient of $1 \mathrm{ps}^{-1}$. All interactions in the simulated systems were described with CHARMM potential. The CHARMM force field includes intramolecular harmonic stretching $V_{\text {bond }}$, harmonic bending $V_{\text {angle }}$, torsional $V_{\text {dihedral }}$ terms

$$
\begin{aligned}
& V_{\text {total }}=V_{\text {bond }}+V_{\text {angle }}+V_{\text {dihedral }} \\
& +V_{v d W}+V_{\text {Coulomb }},
\end{aligned}
$$
where

$$
\begin{aligned}
& V_{\text {bond }}=K_{r}\left(r-r_{0}\right)^{2} \\
& V_{\text {angle }}=K_{\Theta}\left(\Theta-\Theta_{0}\right)^{2} . \\
& V_{\text {dihedral }}= \begin{cases}K_{\phi}(1+\cos (n \phi-\gamma)) & n \neq 0, \\
K_{\phi}(\phi-\gamma)^{2} & n=0,\end{cases} \\
& V_{v d W}=4 \epsilon\left[\left(\frac{\sigma}{r}\right)^{12}-\left(\frac{\sigma}{r}\right)^{6}\right] .
\end{aligned}
$$

The last two terms in equation for the total potential energy $V_{\text {total }}$ of the system, come from the nonbonding interactions: van der Waals forces modeled with the standard Lenard-Jones (12-6) potential $V_{v d W}$ (with the Lorentz-Berthelot mixing rules) and electrostatic interactions $V_{\text {Coulomb. }}$ The TIP3P CHARMM model of water [45] was used in our simulations. The cutoff distance for all non-bonding interactions was set to $10 \AA$. All molecules used in our studies were modeled at the full atomistic level. 


\section{Results}

The initial configuration was chosen as sandwich-like, by placing nine $\beta$-CD molecules between two parallel layers of DMPC. After an equilibration process, the MD simulations were performed and the appropriate data collected. In Fig. $2 \mathrm{a}-\mathrm{f}$ we show the series of snapshots visualizing the transformation of the starting, sandwichlike structure to the final one. The micelles formed from
DMPC molecules are covered by $\beta$-CD molecules. It suggests that the attractive component of DMPC-DMPC interaction overcomes its counterpart in $\beta$-CD-DMPC interacting pair. Hence, the DMPC molecules are "getting together", that leads to formation of the DMPC cluster. The $\beta$-cyclodextrin molecules stick to the surface of the created DMPC cluster. The interaction DMPC $-\beta$-CD is not strong enough to extract the DMPC molecules out of the micelles.
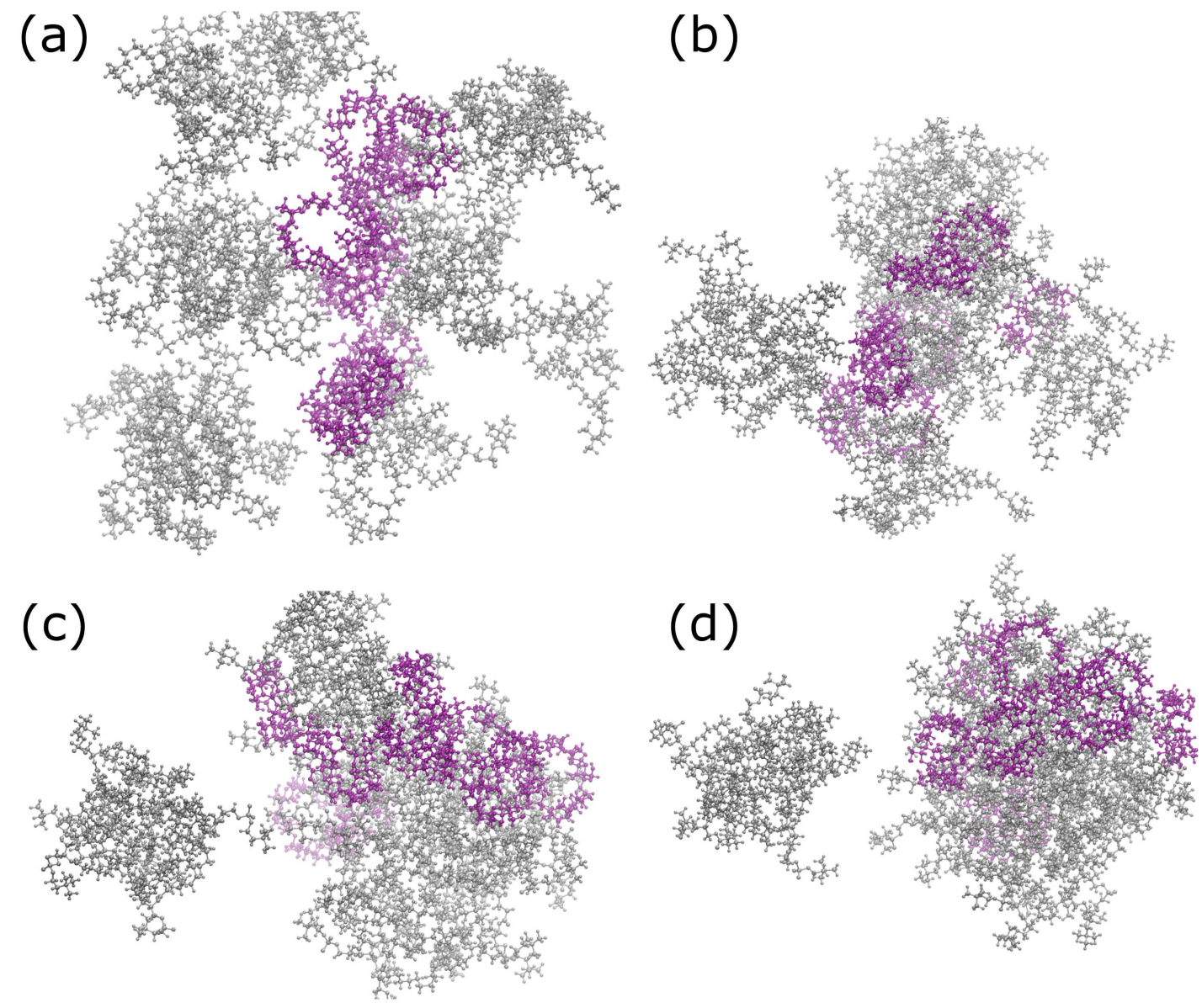

(e)
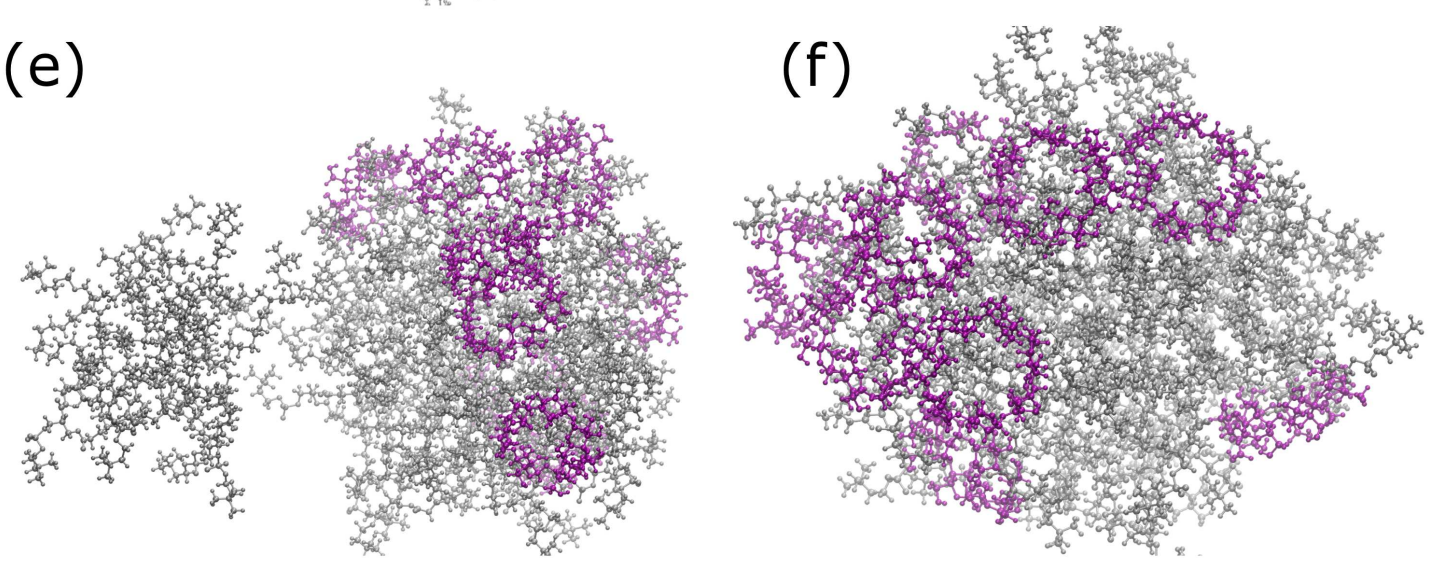

Fig. 2. The sequence of snapshots visualizing the process of transformation from the mixture of $\beta$-CD and DMPC molecules to DMPC micelle surrounded by $\beta$-CD molecules in water environment, at $T=330 \mathrm{~K}$. The water molecules are not shown for clarity. 
We calculated the average number of hydrogen bonds per one $\beta$-CD molecule, for several temperatures. Hydrogen bonds are calculated among $\beta$-CD and water as well as $\beta$-CD and DMPC molecules. A hydrogen bond is formed between an atom with a hydrogen bonded to it (called donor D), and another atom (the acceptor A) provided that the acceptor-donor distance $(\mathrm{D}-\mathrm{A})$ is less than the cut-off distance (default $3.0 \AA$ ) and the angle D-A-H is less than the cut-off angle (default $20^{\circ}$ ). Our calculations have shown that $\beta$-CD molecules have greater affinity for water molecules than for DMPC molecules, forming on average 4.32 bonds with water and 0.01 bonds with DMPC at temperature $T=310 \mathrm{~K}$. Calculated hydrogen bonds and visual inspection of the obtained trajectories revealed that there are no DMPC- $\beta$-CD structures based mainly on hydrogen bonds.

The radial distribution function $g(r)$, calculated between fourth carbon atom from the glucopyranose ring in $\beta C D$ and last carbon atom in hydrophobic tail of

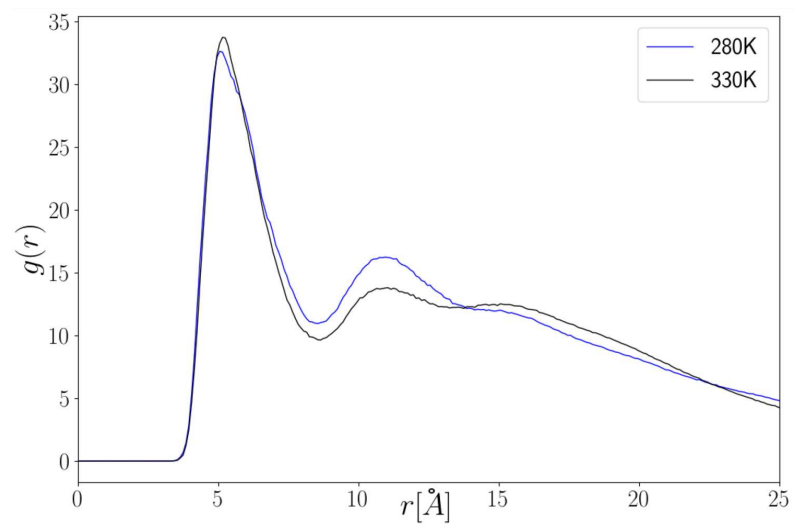

Fig. 3. The radial distribution function between fourth carbon atom from the glucopyranose ring in $\beta$-CD and last carbon atom in hydrophobic tail of DMPC for $\beta$-CD:DMPC ratio 1:2.

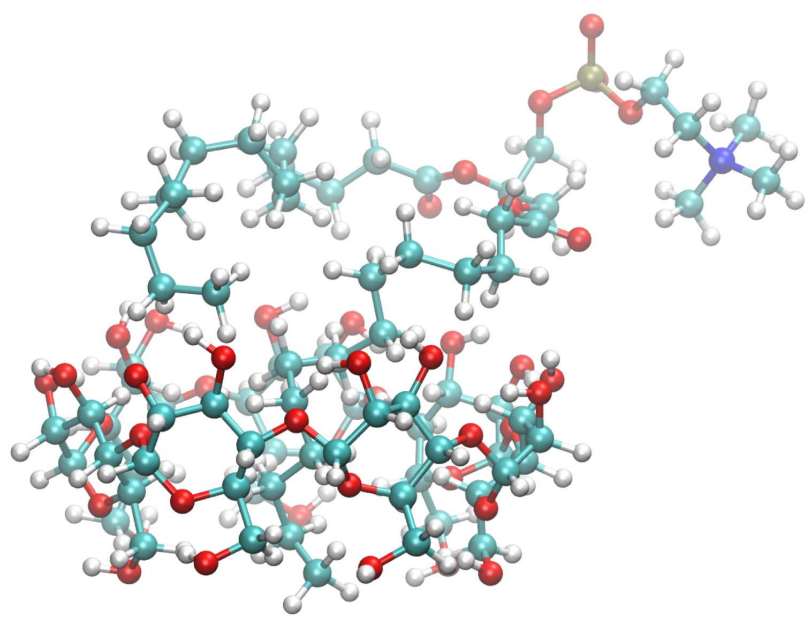

Fig. 4. The structure of complex $\beta$-CD with DMPC.
DMPC is shown in Fig. 3. While the first pick of the radial distribution function remains relatively stable and does not move or significantly diminish with the increase of temperature, it can be observed that the second pick decreases with temperature.

In our computations we observed the formation of DMPC- $\beta-C D$ complex. This complex is the "hostguest" type inclusion compound in which cyclodextrin acts as a "host" and partially hydrophobic species are "guest". The complex was formed by the attraction of hydrophobic hydrocarbon group of the DMPC molecule with the hydrophobic interior of the $\beta$-CD. This type of structure is presented in the Fig. 4. This complex is hydrophilic, water-soluble, and thermodynamically stable. The thermal stability of this complex is additionally confirmed by the aforementioned stability of the first pick of the radial distribution plot.

We have also performed calculations of the root mean square displacement of atomic positions (RMSD) [46] using the VMD RMSD Trajectory Tool [41],

$$
\operatorname{RMSD}(t)=\sqrt{\frac{\sum_{i=1}^{N}\left(\boldsymbol{r}_{i}(t)-\boldsymbol{r}_{i}(0)\right)^{2}}{N}}
$$

where $\boldsymbol{r}_{i}(t)$ is position of $i$-th atom in time $t$.
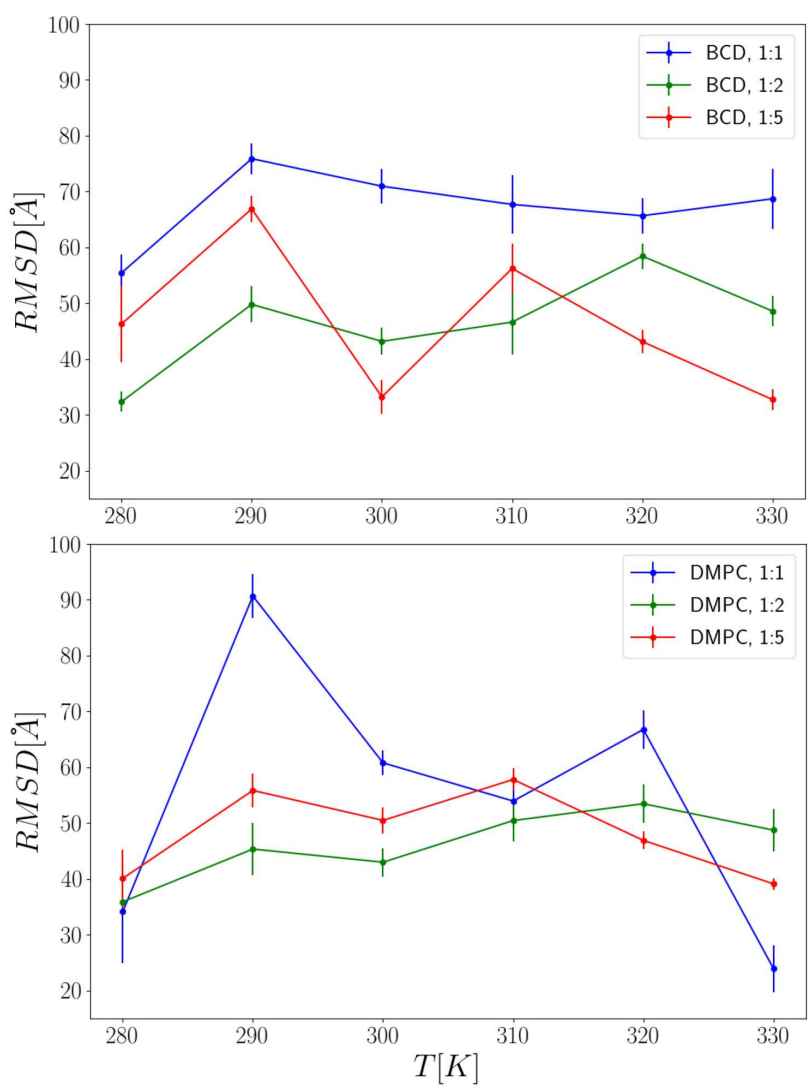

Fig. 5. RMSD as a function of temperature for various $\beta$-CD:DMPC ratios for $\beta$-CD and DMPC molecules. 
These calculations were done separately for DMPC and $\beta$-CD species. The RMSD results are shown in Fig. 5 as a function of temperature, for several ratios $\beta$-CD:DMPC in water environment. The presented RMSD was determined as the average of the course of the root mean square displacement from the final part of the obtained trajectories (form $10 \mathrm{~ns}$ onward). Only the core atoms were taken into account (hydrogens were excluded from the calculations). The obtained results indicate that the mobility of both DMPC and $\beta$-CD molecules only slightly depend on temperature for aqueous systems. The obtained values of RMSD are an order of magnitude higher than typically reported for systems composed of phospholipids [47, 48]. However it should be noted, that in studied systems, the concentration of phospholipids is too low to form a stable lamellar structure, and phospholipids are able to travel in the entire volume of phospholidid $-\beta$-CD cluster.

\section{Conclusions}

We performed a series of MD simulations of binary $\beta$-cyclodextrin-DMPC clusters in the presence of water. In water environment, the DMPC molecules form micelles surrounded by $\beta$-CD molecules. It means that in a water environment the attractive component of DMPC-DMPC interaction prevails its counterpart in $\beta$ CD-DMPC pair. As a result, the DMPC molecules have the tendency to "get together" forming the DMPC cluster (core), whereas $\beta$-cyclodextrin molecules stick to the cluster surface. The DMPC core covered by $\beta$-cyclodextrines is a stable, rather specific aggregate. The developed $\beta$ cyclodextrine shell might facilitate the transport of DMPC core and adaptation in bioenvironment, thus opening the perspective for future applications in biotechnology and nanomedicine. We also found that the alone pair of DMPC and $\beta$ CD can make a molecular complex. Our results suggest that $\beta$-cyclodextrine does not affect the structure of lipde aggregates and should not negatively impact the liposomal drug delivery systems. Finally note, that we made our MD simulations for the molecular complex $\beta$ CD-DMPC, surrounded only by the water molecules. This is a common procedure, since water is an essential component of a typical bio sample. However, our reconnaissance study presented here, can be in future extended towards more specific bio environments. Then, in addition to water one should include in MD simulations also other components of a particular bioenvironment of interest, for example, sodium and chloride ions in case of physiological fluid (saline).

\section{Acknowledgments}

This research was supported in part by PAAD Infrastructure co-financed by Operational Programme Innovative Economy, Objective 2.3.

\section{References}

[1] H. Abramczyk, A. Imiela, A. Sliwinska, J. Mol. Liq. $\mathbf{2 7 4}, 52$ (2019).

[2] H. Abramczyk, B. Brozek-Pluska, M. Krzesniak, M. Kopec, A. Morawiec-Sztandera, Spectrochim. Acta A 129, 609 (2014).

[4] P. Raczyński, V. Raczyńska, K. Górny, Z. Gburski, Arch. Biochem. Biophys. 580, 22 (2015).

[5] A. Piatek, A. Dawid, Z. Gburski, Spectrochim. Acta A 79, 819 (2011).

[6] P. Raczyński, A. Dawid, Z. Gburski, J. Mol. Struct. 792, 212-215 (2006).

[7] A. Kachel, Z. Gburski, J. Phys. 9, 10095 (1997).

[8] S. Pal, S. Balasubramanian, B. Bagchi, J. Phys. Chem. B 107(22), 5194 (2003).

[9] H. Fathi, J.P. Kelly, V.R. Vasquez, O.A. Graeve, Langmuir 28, 9267 (2012).

[10] R. L. Mancera, M. Chalaris, K. Refson, J. Samios, Phys. Chem. Chem. Phys. 6, 94 (2004).

[11] M. Schlenkrich, J. Brickmann, A.D. MacKerell, M. Karplus, in: Biological Membranes, Springer, 1996, pp. 31-81.

[12] S.J. Marrink, D.P. Tieleman, A.E. Mark, J. Phys. Chem. B 104, 12165 (2000).

[13] S. Abel, F.Y. Dupradeau, E.P. Raman, A.D. MacKerell Jr., M. Marchi, J. Phys. Chem. B 115, 487 (2010).

[14] S. Abel, F.Y. Dupradeau, M. Marchi, J. Chem. Theory Comput. 8, 4610 (2012).

[15] S.A. Sanders, M. Sammalkorpi, A.Z. Panagiotopoulos, J. Phys. Chem. B 116, 2430 (2012).

[16] T. Mizuguchi, R. Ishizuka, N. Matubayasi, Chem. Phys. Lett. 624, 19 (2015).

[17] A.P. Santos A.Z. Panagiotopoulos, J. Chem. Phys. 144(4), 044709 (2016)

[18] S. Faramarzi, B.Bonnett, C. A. Scaggs, A. Hoffmaster, D. Grodi, E. Harvey, B. Mertz, Langmuir 33, 9934 (2017)

[19] K. Smalara, A. Giełdon, M. Bobrowski, J. Rybicki, C. Czaplewski, J. Phys. Chem. A 114, 4296 (2010).

[20] P. Alejster, W. Jurkowski, I. Roterman-Konieczna, in: Protein Folding in Silico, Elsevier, 2012, p. 39.

[21] W. Chmielnicki, I. Roterman-Konieczna, K. Stapor, Expert Systems 29, 200 (2012).

[22] D. Makieła, I. Janus-Zygmunt, K. Górny, Z. Gburski, J. Mol. Liq. 262, 451 (2018).

[23] B. Padlyak, S. Mudry, V. Halchak, A. Korolyshyn, J. Rybicki, A. Witkowska, Opt. Appl. 30, 691 (2000).

[24] S. Pawar, P. Shende, F. Trotta, Int. J. Pharm. 565, 333 (2019).

[25] P.P. Menezes, T.A. Andrade, L.A. Frank, E.P. Souza, G.G.G. Trindade, I.A.S. Trindade, M.R. Serafini, S.S. Guterres, A.A.S. Araújo, Int. J. Pharm. 559, 312 (2019).

[26] A. Di Cicco, M. Minicucci, E. Principi, A. Witkowska, J. Rybicki, R. Laskowski, J. Phys. Condens. Matter 14(12), 3365 (2002).

[27] D. Makieła, I. J. Zygmunt, K. Górny, Z. Gburski, J. Mol. Liq. 288, 110974 (2019). 
[28] L. Szente E. Fenyvesi, Struct. Chem. 28, 479 (2017).

[29] M. Roux, R. Auzely-Velty, F. Djedaini-Pilard, B. Perly, Biophys. J. 82, 813 (2002).

[30] M.K. Grachev, M.A. Malenkovskaya, L.K. Vasyanina, J. Incl. Phenom. Macro. Chem. 83, 209 (2015).

[31] J.P. Litz, N. Thakkar, T. Portet, S.L. Keller, Biophys. J. 110, 635 (2016).

[32] A. Joset, A. Grammenos, M. Hoebeke, B. Leyh, J. Incl. Phenom. Macro. Chem. 83, 227 (2015).

[33] J. Mascetti, S. Castano, D. Cavagnat, B. Desbat, Langmuir 24, 9616 (2008).

[34] A. Tsamaloukas, H. Szadkowska, P.J. Slotte, H. Heerklotz, Biophys. J. 89, 1109 (2005).

[35] J. Zhang, P.X. Ma, Adv. Drug Deliv. Rev. 65, 1215 (2013).

[36] O. Adeoye, H. Cabral-Marques, Int. J. Pharm. 531, 521 (2017).

[37] A. Dawid, Z. Gburski, J. Mol. Struct. 482, 271 (1999).

[38] A. Dawid, Z. Gburski, J. Phys. Condens. Matter 15, 2399 (2003)

[39] J.C. Phillips, R. Braun, W. Wang, J. Gumbart, E. Tajkhorshid, E. Villa, C. Chipot, R.D. Skeel, L. Kalé, K. Schulten J. Comput. Chem. 26, 1781 (2005).
[40] B.R. Brooks, C.L. Brooks, A.D. Mackerell, L. Nilsson, R.J. Petrella, B. Roux, Y. Won, G. Archontis, C. Bartels, et al. J. Comput. Chem. 30, 1545 (2009)

[41] W. Humphrey, A. Dalke, K. Schulten, J. Mol. Graph. 14, 33 (1996).

[42] W.G. Noid, J. Chem. Phys. 139, 090901 (2013).

[43] T. Darden, D. York, L. Pedersen, J. Chem. Phys. 98(12), 10089 (1993)

[44] A. Brünger, C.L. Brooks, M. Karplus, Chem. Phys. Lett. 105, 495 (1984)

[45] W.L. Jorgensen, J. Chandrasekhar, J.D. Madura, R.W. Impey, M.L. Klein, J. Chem. Phys. 79, 926 (1983).

[46] E.A. Coutsias, C. Seok, K.A. Dill, J. Comput. Chem. 25, 1849 (2004).

[47] A.L. Rabinovich, P.O. Ripatti, N.K. Balabaev, F.A.M. Leermakers, Phys. Rev. E 67, 011909 (2003).

[48] A.Y. Shih, I.G. Denisov, J.C. Phillips, S.G. Sligar, K. Schulten, Biophys. J., 88, 548 (2005). 